\section{Antiviral Medication Use and Cost of Hepatitis Infected Diseases among 105 Hospitals in China from 2009 to 2013}

\section{Abstract}

Background: Chronic hepatitis is a major health problem that can lead to decompensated cirrhosis, hepatocellular carcinoma, and, eventually death, all of which are associated with significant healthcare costs.

Objective: The purpose of this study was to assess the use of antivirals among hospitals in Shanghai, China, between 2009 and 2013.

Methods: A retrospective analysis was performed on the main types and manufacturers, dosages, defined daily dosages (DDDs), sales volumes, and proportions of total sales of antivirals among 105 sample hospitals in Shanghai, China, from 2009 to 2013.

Results: During 2009 to 2013, antivirals experienced rapid growth rates in sales compared to the growth rates of sales of all drugs combined (2009: $34.92 \%$ vs. $21.31 \%$; 2010 : $12.35 \%$ vs. $10.63 \%$; 2011 : $22.51 \%$ vs. $9.47 \%$; 2012 : $18.8 \%$ vs $10.98 \%$; 2013: $11.15 \%$ vs. $8.57 \%$ ). Although the proportion of all drugs that were antivirals was small, the proportion increased each year, from 1\% of all purchases in 2009 to $1.10 \%$ in $2010,1.23 \%$ in $2011,1.57 \%$ in 2012 , and $1.89 \%$ in 2013 .

Conclusion: Sales of anti-hepatitis B virus (HBV) nucleoside drugs, such as lamivudine, entecavir, adefovir dipivoxil, and telbivudine, showed rapid upward trends. These drugs occupied the top rankings in terms of sales volume and dosage among the 105 sample hospitals. The antiherpes drugs valaciclovir and ganciclovir also showed upward trends, whereas stavudine, acyclovir, and penciclovir showed downward trends. The use of antiviral medications, especially those for HBV, in Shanghai, China, has shown consistently strong growth in terms of sales and dosage.

Keywords: Antiviral; Analysis of drug use; Nucleoside; $D^{2}$; Clinical evaluation

\section{Jing Tang ${ }^{1}$, Yue Liang ${ }^{2}$, Gende Li ${ }^{3}$, Weifeng Shi ${ }^{1}$ and Cheng Gui ${ }^{1}$}

1 Department of Clinical Pharmacy, School of Medicine, Shanghai First People's Hospital, Shanghai Jiaotong University, Shanghai 201620, China

2 Department of Pharmacy, Peking University Shenzhen Hospital, Shenzhen 518030, China

3 Department of Pharmacy, Dehong Prefecture of Yun'nan Province People's Hospital, Dehong 678400, China

Corresponding author: Dr. Jing Tang angel_tj99@126.com

Tel: $86-02137798317$

Fax: 86-02137798605

Department of Clinical Pharmacy, School of Medicine, Shanghai First People's Hospital, Shanghai Jiaotong University, No. 650 New Songjiang Road, Shanghai 201620, China.

\author{
Citation: Tang J, Liang Y, Li G, et al. \\ Antiviral Medication Use and Cost of \\ Hepatitis Infected Diseases among 105 \\ Hospitals in China from 2009 to 2013. \\ J Infec Dis Treat. 2016, 2:1.
}

\section{Introduction}

As an infectious particle without a cell structure but with its own enzymatic system, a virus requires a host cell for replication. Viruses may invade the tissues and organs of the body and infect the cells to cause disease. Common diseases caused by viruses include epidemic diseases, such as influenza, rhinovirus, measles, mumps, polio, and infectious hepatitis, as well as chronic diseases, such as hepatitis $B$ and acquired immunodeficiency syndrome (AIDS) related to human immunodeficiency virus (HIV). Other common virus-caused diseases are latent infections, herpetic keratitis, sexually transmitted herpes disease, and certain tumors.
Due to their parasitic nature, viruses are not easy to treat clinically. Antiviral medications (antivirals) are a cost-effective method to reduce viral replication, and their use may decrease liver complications, hospitalizations, and mortality [1,2]. However, very few antivirals are clinically effective for the long-term treatment of viral diseases. With the recent, rapid, and global spread of viral diseases such as influenza, viral hepatitis, and AIDS, the demand for antivirals has sharply increased, promoting the development of new drugs.

An estimated 120 million people are infected with hepatitis $B$ virus (HBV) in China, which accounts for about $1 / 3$ of the total 
global hepatitis B population. Each year, nearly 50 million people die from HBV-induced liver damage and liver cancer. Therefore, the early standardized use of antiviral therapy is essential. To evaluate antiviral medication usage in China, we conducted a large, retrospective analysis of antiviral purchases by 105 sample hospitals in Shanghai, China, from 2009 to 2013.

\section{Methods}

Data regarding antiviral purchases that were reported to the Science and Technology Information Institute of the Shanghai Food and Drug Administration (SFDA) were obtained for 105 sample hospitals in Shanghai, China, for the period from 2009 to 2013. The 105 sample hospitals included 29 tertiary, 64 secondary, and 12 primary hospitals. The antiviral purchase data included the drug name (generic and trade names), formulation, and manufacturer. A statistical analysis was performed on the antiviral data from the sample hospitals. The analyzed variables included the sales volume of antivirals, the sales growth rate of antivirals, the proportion of total medication purchases that were antivirals, the frequency of antiviral medication (in terms of defined daily dose, DDD), and the type of antiviral. The DDDs for different antivirals, which can be found on the package inserts for medications, have been compiled by the Shanghai Pharmaceutical Society in their Clinical Utility of New Drugs Manual (17 ${ }^{\text {th }}$ Edition). In the present study, the DDDs were taken from this manual. The DDD reflects the quality of the drug in its different formulations and can be used to compare drug usage between brands or healthcare environments.

\section{Results}

Table 1 shows the results of purchases of antiviral medications by the 105 sample hospitals in Shanghai, China, between 2009 and 2013. Sales growth rates of antivirals slowed over this time period. Hospitals spent 1.37 times the amount of money on antiviral medications in 2013 as they did in 2009. The proportion of antivirals is increasing each year.

Tables 2 and $\mathbf{3}$ show the sales volumes of the major types of antivirals for the 105 sample hospitals in Shanghai, China, from 2009 to 2013. The sales of anti-HBV nucleoside drugs rose rapidly among the hospitals from 2009 to 2013, with the top 3 antiviral medications in terms of sales volume being entecavir, lamivudine, and adefovir dipivoxil. The top 3 antivirals in terms of dosage were lamivudine, entecavir, and adefovir dipivoxil.

Table 4 shows the top 10 leading manufacturers of major antivirals purchased by the sample hospitals from 2009 to 2013. The top 3 manufacturers of antivirals in terms of sales volume

Table 1 Antiviral and total drug purchases among 105 sample hospitals in Shanghai, China, from 2009 to 2013.

\begin{tabular}{|cc|}
\hline Year & $\begin{array}{c}\text { Antiviral drugs } \\
\text { Growth rate (\%) }\end{array}$ \\
\hline 2009 & 34.92 \\
2010 & 12.35 \\
2011 & 22.51 \\
\hline 2012 & 18.80 \\
\hline 2013 & 11.15 \\
\hline
\end{tabular}

Table 2 Top 20 antivirals in terms of sales volume among 105 sample hospitals in Shanghai, China, from 2009 to 2013.

\begin{tabular}{|c|c|c|c|c|c|}
\hline Drug name & \multicolumn{6}{|c|}{ Growth rate (\%) } \\
\hline Entecavir & $\mathbf{2 0 0 9}$ & $\mathbf{2 0 1 0}$ & $\mathbf{2 0 1 1}$ & $\mathbf{2 0 1 2}$ & $\mathbf{2 0 1 3}$ \\
\hline Lamivudine & $\mathbf{5 3 . 6 5}$ & 22.69 & 31.91 & 40.15 & 39.41 \\
\hline Adefovir dipivoxil & $\mathbf{2 8 . 0 3}$ & 13.25 & 7.63 & 5.14 & 4.77 \\
\hline Telbivudine & 68.65 & -4.14 & 63.47 & 51.14 & 40.14 \\
\hline
\end{tabular}

Note: Growth rate (\%) is compared with the previous year.

from 2009 to 2013 were joint ventures or foreign manufacturers: GlaxoSmithKline (China) Investment Co., Ltd., Sino-America Shanghai Squibb Pharmaceuticals Ltd., and Beijing Novartis Pharma Ltd. Domestic pharmaceutical companies in China included Sinopharm Chuan Kang Pharmaceutical Co., Ltd., Jiangsu Chia-Tai Tianqing Pharmacy Co., Ltd., Livzon Pharmaceutical Group Inc., Hubei Keyi Pharmaceutical Co., Ltd., and Shanghai Sine Pharmaceutical Co., Ltd.. Among the domestic manufacturers, Baraclude of Sino-America Shanghai Squibb Pharmaceuticals Ltd., Heptodin of Beijing Novartis Pharma Ltd. occupied major antiviral markets in the Shanghai hospitals.

\section{Discussion}

Anti-HBV medications accounted for a large proportion of the antivirals showing fast sales growth in recent years. As the incidence of chronic hepatitis $B$ has risen, so has the rate at which the National Medical Insurance System has covered its treatment, leading to an increase in drug demand. Food and Drug Administration (FDA)-approved treatments for HBV include lamivudine, adefovir dipivoxil and telbivudine. These drugs act on the HBV polymerase gene (P gene) to inhibit HBV DNA replication directly. Treatment with these drugs leads to improved liver histology, delays the progression of hepatitis $B$, and reduces the incidence of cirrhosis and liver cancer [1].

In the 1990s, lamivudine was released to the domestic market as the first nucleoside treatment for chronic hepatitis B. However, long-term lamivudine use is associated with viral gene mutations. As time on the medication increases, the proportion of patients with viral mutations also increases, from $14 \%$ in the first year to $38 \%, 49 \%$, and $66 \%$ in the second, third, and fourth year, respectively. The high mutation rate leads to drug resistance, which limits the long-term application of this medication [3]. Nevertheless, because of its rapid inhibition of HBV, relatively few toxic side effects, and low price, lamivudine is frequently used as an early treatment for hepatitis $B$. In recent years, the dosages of lamivudine used by the sample hospitals in Shanghai tended to increase. The sales growth showed an increase of $27.02 \%$ from 2007 to 2008 and an increase of $28.03 \%$ from 2008 to 2009 (data not shown). Lamivudine retained the first place position in terms of DDDs ranking during 2009-2013.

The antiviral capacity of adefovir dipivoxil is not stronger than that of lamivudine and, in large doses, adefovir dipivoxil can cause renal toxicity. However, adefovir dipivoxil is useful in patients who are resistant to lamivudine. Although patients can become resistant to adefovir dipivoxil, the time frame of resistance is much longer than that for lamivudine because the virus mutates 
Table 3 Dosage amounts and defined daily dosages (DDDs) of major antivirals among 105 sample hospitals in Shanghai, China, from 2009 to 2013.

\begin{tabular}{|c|c|c|c|c|c|c|c|c|c|c|c|}
\hline \multirow[b]{2}{*}{ Drug name } & \multirow[b]{2}{*}{ DDD } & \multicolumn{2}{|c|}{2009} & \multicolumn{2}{|c|}{2010} & \multicolumn{2}{|c|}{2011} & \multicolumn{2}{|c|}{2012} & \multicolumn{2}{|c|}{2013} \\
\hline & & Dosage (g) & $\begin{array}{c}\mathrm{DDD}_{\mathrm{s}} \\
\text { Ranking }\end{array}$ & Dosage (g) & $\begin{array}{c}\mathrm{DDD}_{\mathrm{s}} \\
\text { Ranking }\end{array}$ & Dosage (g) & $\begin{array}{c}\mathrm{DDD}_{\mathrm{s}} \\
\text { Ranking }\end{array}$ & Dosage (g) & $\begin{array}{c}\mathrm{DDD}_{\mathrm{s}} \\
\text { Ranking }\end{array}$ & Dosage (g) & $\begin{array}{c}\mathrm{DDD}_{\mathrm{s}} \\
\text { Ranking }\end{array}$ \\
\hline Lamivudine & $0.1 \mathrm{~g}$ & 314000 & 1 & 355303 & 1 & 399358 & 1 & 411576 & 1 & 465752 & 1 \\
\hline Entecavir & $0.5 \mathrm{mg}$ & 925 & 2 & 891 & 2 & 1644 & 2 & 2044 & 2 & 2471 & 2 \\
\hline $\begin{array}{l}\text { Adefovir } \\
\text { dipivoxil }\end{array}$ & $0.01 \mathrm{~g}$ & 14600 & 3 & 14302 & 3 & 24214 & 3 & 28741 & 3 & 31402 & 3 \\
\hline Telbivudine & $0.6 \mathrm{~g}$ & 240000 & 4 & 239743 & 4 & 309064 & 4 & 387410 & 4 & 451024 & 4 \\
\hline
\end{tabular}

Table 4 Top 10 leading manufacturers of antivirals among 105 sample hospitals in Shanghai, China, from 2009 to 2013.

\begin{tabular}{|c|c|c|c|}
\hline Manufacturer & Trade name (Generic Name) & Ranking (2009-2013) \\
Sino-America Shanghai Squibb Pharmaceuticals Ltd. & Baraclude (Entecavir) & 1 \\
\hline GlaxoSmithKline (China) Investment Co. Ltd. & Heptodin (Lamivudine), & 2 \\
Beijing Novartis Pharma Ltd. & Hepsera (Adefovir dipivoxil) & 3 \\
\hline Jiangsu Chia-Tai Tianqing Pharmacy Co. Ltd. & Sebivo (Telbivudine) & 4 \\
Shanghai Yishengyuan Pharmaceutical Co. Ltd. & Adefovir dipivoxil & 5 \\
\hline
\end{tabular}

much later. One study showed that the incidence rates of viral mutations with adefovir dipivoxil administration during the first through fifth years were $0 \%, 3 \%, 11 \%, 18 \%$, and $29 \%$, respectively [4]. In hospitals in Shanghai, adefovir dipivoxil was mainly used for lamivudine-resistant patients. This antiviral showed increasing sales volumes over the past 5 years, with a third-place ranking in terms of DDDs in 2009.

Entecavir has been available on China's market since early 2006. Entecavir has strong anti-HBV effects, and a low incidence of resistance, with cumulative resistance rates in naive patients of $0.2 \%, 0.5 \%$, and $1.2 \%$ after 1,2 , and 3 years, respectively $[5,6]$. The main disadvantage of this drug is that it is expensive. The sales volume of entecavir has increased significantly in Shanghai hospitals since 2009, even more so than lamivudine. Entecavir ranked first in terms of sales volume and second in the DDDs ranking among the 105 sample hospitals. However, more clinical observation time of this drug is needed.

Telbivudine is another anti-HBV medication approved for domestic use. Telbivudine has been reported to show superior antiviral activity and clinical efficacy compared to lamivudine [7]. In October, 2006, telbivudine was approved for use by the FDA. Since it was listed in the SFDA in February, 2007, usage of the drug among hospitals in Shanghai has increased sharply. Telbivudine is the only pregnancy class $B$ antiviral drug to be approved by the FDA (lamivudine, adefovir dipivoxil, and entecavir are pregnancy class C). However, with such a short time on the market and expensive treatment cost, the prospects for telbivudine use await further study $[8,9]$.

\section{Conclusion}

In this study, among the 105 sample hospitals in Shanghai that were studied, antivirals demonstrated rapid growth in some varieties. The amount of anti-HBV drugs accounted for more than $85 \%$ of all antiviral drugs and its use continues to increase. In terms of the amount of sales of antivirals from the top 10 pharmaceutical companies, foreign investment and joint venture products occupied most of the market, with the market share of domestic enterprises gradually increasing.

\section{Acknowledgments and Funding}

This study was supported by the Natural Science Foundation of China (No. 81470852), the Science and Technology Commission of Shanghai Science and Technology Support Project (No. 13431900503), the Medical and Technology across Project of Shanghai Jiao Tong University (No. YG2012MS02), and the Young Talents Plan of Shanghai Health System (No. XYQ2013091). 


\section{References}

1 Barr CE, Schulman K, lacuzio D (2007) Effect of oseltamivir on the risk of pneumonia and use of health care services in children with clinically diagnosed influenza. Curr Med Res Opin 23: 523-531.

2 McGeer A, Green KA, Plevneshi A (2007) Antiviral therapy and outcomes of influenza requiring hospitalization in Ontario, Canada. Clin Infect Dis 45: 1568-1575.

3 Perrillo RP (1993) Antiviral therapy of chronic hepatitis B: past, present, and future. J Hepatol 17: S56-S63.

4 European Association for the Study of the Liver (2012) EASL clinical practice guidelines: Management of chronic hepatitis B virus infection. J Hepatol 57: 167-185.
5 Miyauchi T, Kanda T, Shinozaki M (2013) Efficacy of Lamivudine or Entecavir against Virological Rebound after Achieving HBV DNA Negativity in Chronic Hepatitis B Patients. Int J Med Sci 10: 647-652.

6 Kim KH, Kim ND, Seong BL (2010) Discovery and development of anti-HBV agents and their resistance. Molecules 15: 5878-5908.

7 Palumbo E (2008) New drugs for chronic hepatitis B: a review. Am J Ther 15: 167-172.

8 Shun-Shin M, Thompson M, Heneghan C (2009) Neuraminidase inhibitors for treatment and prophylaxis of influenza in children: systematic review and meta-analysis of randomised controlled trials. BMJ 339: b3172.

9 Xu J, Lu ZM (2006) Progress in the treatment of chronic hepatitis B with Telbivudine. Zhonghua Gan Zang Bing Za Zhi 14: 958-960. 